\title{
Efectos de aplicar la gestión de ingresos en la industria aeronáutica
}

Effects of the application of Revenue Management in the aeronautical industry

\author{
Daniela Álvarez ${ }^{1}$ Renato Quiliche ${ }^{2}$ Mario Chong ${ }^{3}$
}

Recibido: 08/2018

Aceptado 09/2019

Resumen. - Las aerolíneas alrededor del mundo se han visto afectadas por los desafíos de rentabilidad, participación de mercado y el comportamiento de la demanda, lo cual ha contribuido al desarrollo de la gestión de ingresos o Revenue Management. El modelo de gestión busca maximizar el nivel de ingreso en las empresas con la gestión de la demanda. La aplicación de este modelo en empresas de diversos rubros ha generado un incremento en sus ingresos anuales en orden del 4 a $5 \%$ favoreciendo oportunidades de negocio a través del desarrollo de nuevos mercados basados en la rentabilidad empresarial. La industria aeronáutica fue una de las pioneras en aplicar este modelo de gestión a nivel mundial. En esta oportunidad, el caso aplica el modelo en un mercado dinámico y con nuevas restricciones: mercado regional, vuelos cortos, tarifas desreguladas e impacto de las aerolíneas de bajo costo.

Palabras clave: Gestión de ingresos, rentabilidad, competencia.

Abstract. -Airlines around the world have been affected by the challenges of profitability, market share and demand behavior, which has contributed to the development of revenue management or Revenue Management. The management model seeks to maximize the level of income in companies with demand management. The application of this model in companies of various sectors has generated an increase in their annual income in order of 4 to $5 \%$ favoring business opportunities through the development of new markets based on business profitability. The aviation industry was one of the pioneers in applying this management model worldwide. On this occasion, the case applies the model in a dynamic market with new restrictions: regional market, short flights, deregulated fares and impact of low-cost airlines.

Keywords: Revenue management, profitability, competition

\footnotetext{
${ }^{1}$ Universidad del Pacífico, alvarezada@ @alum.up.edu.pe . ORCID 0000-0002-4846-2605

2 Universidad del Pacífico r.quilichealtamirano@up.edu.pe . ORCID 0000-0001-7937-5929

3 Universidad del Pacífico, m.chong@up.edu.pe . ORCID 0000-0002-1231-0992

Memoria Investigaciones en Ingeniería, 2019. $\mathrm{N}^{\circ} 17(1)$. pp 61-71

https://doi.org/10.36561/ING.17.4

ISSN 2301-1092 • ISSN (en línea) 2301-1106
} 
1. Introducción. El reporte de la Asociación Internacional de Transporte Aéreo (IATA por sus siglas en inglés) de 2016 [1] presentó la influencia del transporte aéreo en la economía global y su impacto en el desarrollo de las comunidades, por la dinámica en la conexión entre ciudades, el intercambio comercial, la generación de empleo, el turismo y las inversiones directas. Sin embargo, las diversas aerolíneas alrededor del mundo han enfrentado problemas relacionados a rentabilidad, participación en el mercado, demanda fluctuante y aparición de las aerolíneas de bajo costo (low cost).

En el caso del Perú, el transporte aéreo tiene un rol significativo para el desarrollo de las comunidades y el intercambio comercial de productos diferenciados y valorados en los mercados globales [1], todo esto debido a la diversidad, agreste geográfica y al centralismo de su capital, Lima. Esta industria genera en este contexto aproximadamente 4,4 mil millones de Dólares Americanos al año, con una participación del 2,1\% del Producto Bruto Interno (PBI) del país. Esta industria participa en un mercado de competencia monopolística de características oligopólicas, con actores nacionales e internacionales, mercado competitivo, con tasas de crecimiento atractivas y enfocado en el precio, así como en la promoción. Las estrategias enfocadas en costo han generado una coyuntura interesante, en un entorno logístico basado principalmente en transporte terrestre. A pesar del entorno, en un país de casi 33 millones de habitantes, se generó un flujo interno de más de 11 millones de pasajeros en el año 2017, con un aumento del 8,5\% con referencia al año anterior [3]. La participación en el mercado interno está dominada por Aerolínea 1, por sus precios, destinos y frecuencias, frente a los actores presentados en la Figura I.

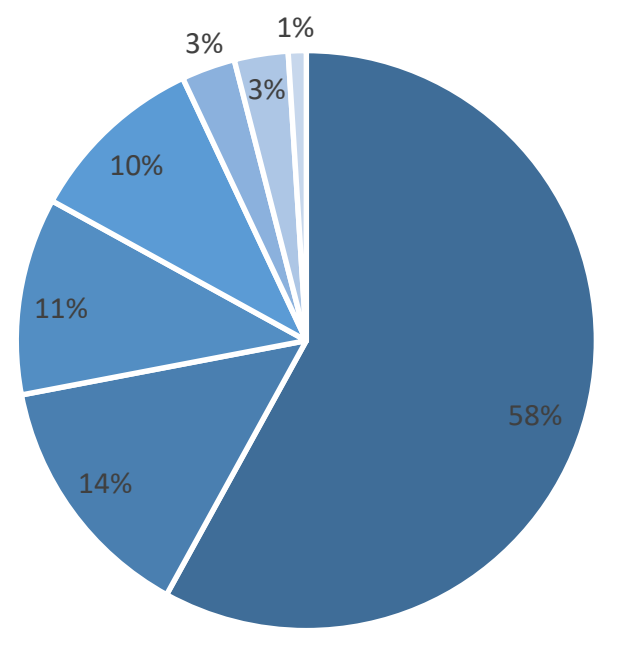

- Aerolínea 1

- Aerolínea 2

- Aerolínea 3

- Aerolínea 4

- Aerolínea 5

- Aerolínea 6

$\backsim$ Otros

Figura I: Participación de mercado de Aerolíneas que transportan pasajeros comerciales en Perú en el 2017. Fuente: Elaboración propia

Una característica de este mercado es la competencia abierta a los destinos para todas las aerolíneas, estas coinciden en la mayoría de los casos en los puntos de origen y destino de los pasajeros locales es (O-D), como se muestra en la matriz a nivel nacional de la Figura II. Bajo este escenario de competencia el ingreso para nuevas aerolíneas no es fácil enfrentar - lograr ser competitivas- manteniendo dos estrategias genéricas, costo y diferenciación. La estrategia de diferenciación se basa en la construcción de una experiencia para el pasajero; a través de una variedad de productos y servicios relacionados como hoteles por perfil de usuario, renta de servicios, visitas especializadas, programas de viajeros frecuentes; enfocado en el objetivo principal, el transporte desde 
el origen al destino. Además, la facilidad para complementar con servicios para cambios de fechas o devoluciones de la compra, tipo de equipajes, considerando el origen y destino principal.

\begin{tabular}{|c|c|c|c|c|c|c|c|}
\hline A & $\begin{array}{c}\text { DISTANCIA } \\
\text { Km. }\end{array}$ & \multicolumn{7}{|c|}{ AEROLÍNEAS } \\
\hline $\mathrm{C} 1<>\mathrm{C} 2$ & 572 & 1 & 2 & 3 & 4 & 5 & 6 \\
\hline $\mathrm{C} 1<>\mathrm{C} 3$ & 764 & 1 & & 3 & 4 & 5 & \\
\hline $\mathrm{C} 1<>\mathrm{C} 3$ & 1010 & 1 & 2 & 3 & & 5 & 6 \\
\hline $\mathrm{C} 1<>\mathrm{C} 4$ & 857 & 1 & 2 & 3 & & 5 & \\
\hline $\mathrm{C} 1<>\mathrm{C} 5$ & 624 & 1 & & 3 & & 5 & 6 \\
\hline $\mathrm{C} 1<>\mathrm{C} 6$ & 488 & 1 & 2 & & 4 & & \\
\hline $\mathrm{C} 1<>\mathrm{C} 7$ & 489 & 1 & & 3 & 4 & & 6 \\
\hline $\mathrm{C} 1<>\mathrm{C} 8$ & 661 & 1 & & & 4 & & \\
\hline $\mathrm{C} 1<>\mathrm{C} 9$ & 837 & 1 & 2 & & & & \\
\hline $\mathrm{C} 1<>\mathrm{C} 10$ & 983 & 1 & & 3 & & & \\
\hline $\mathrm{C} 1<>\mathrm{C} 11$ & 565 & 1 & & & 4 & & 6 \\
\hline $\mathrm{C} 1<>\mathrm{C} 12$ & 1565 & 1 & 2 & & & & \\
\hline $\mathrm{C} 1<>\mathrm{C} 13$ & 328 & 1 & & & 4 & & \\
\hline $\mathrm{C} 1<>\mathrm{C} 14$ & 1014 & 1 & & & & & \\
\hline $\mathrm{C} 1<>\mathrm{C} 15$ & 169 & 1 & & 3 & 4 & & \\
\hline $\mathrm{C} 1<>\mathrm{C} 16$ & 951 & 1 & & & & & \\
\hline $\mathrm{C} 1<>\mathrm{C} 17$ & 730 & 1 & & & & & \\
\hline F & & & & & & & \\
\hline
\end{tabular}

Figura II: Operadores de rutas del doméstico Perú. Fuente: Elaboración propia

Respecto a la competitividad de las aerolíneas: los costos de operación establecidos por las horas de vuelo efectivo y la aparición en el mercado de aerolíneas de bajo costo genera un desafío constante. El ingreso de aerolíneas de bajo costo origina un movimiento interesante en la industria aeronáutica [22] debido al incremento en la oferta de producto y el modelo simplificado en estructura tarifaria. El equilibrio alcanzado en la industria pierde armonía con el ingreso de aerolíneas de bajo costo. La diferencia principal entre ambas propuestas de aerolíneas (tradicionales y de bajo costo) está centrada en la flexibilidad de servicios como reprogramar y cancelar vuelos, así como los productos y servicios ofrecidos durante el vuelo. La tabla I, muestra un comparativo de tarifas entre ambos tipos de aerolíneas. En consecuencia, como respuesta a esta nueva condición en el mercado, algunas aerolíneas han optado por optimizar sus ingresos mediante la gestión de la demanda conocida como "Revenue Management" (RM por sus siglas en inglés), la cual tiene como objetivo alinear demanda y oferta considerando tres dimensiones: 1) producto, en este caso el pasaje aéreo; 2) tipo de cliente y preferencias; y 3) tiempo programado de servicio. El manejo de las tres dimensiones con la gestión de ingresos se ha vuelto prioridad en la industria por el efecto en el aumento de ingresos entre $4 \%$ a $5 \%$ anual en las operaciones. Una de las consideraciones es eliminar la regulación tarifaria de mínima estadía, la cual permite viajes sólo de ida sin requerir regresar. A través de este método de apalancamiento se espera capturar pasajeros ofreciendo vuelos más flexibles. 


\begin{tabular}{|c|c|c|}
\hline Regulación & Aerolíneas de bajo costo & $\begin{array}{c}\text { Aerolínea de modelo } \\
\text { tradicional }\end{array}$ \\
\hline Estadía & $\begin{array}{c}\text { No tiene mínimos de } \\
\text { estadía }\end{array}$ & Maneja mínimos de estadía \\
\hline Anticipación & Sí regula por anticipación & Sí regula por anticipación \\
\hline Cambios de fecha & Sí permite con penalidad & $\begin{array}{c}\text { Permite sólo en tarifas altas y } \\
\text { con penalidad }\end{array}$ \\
\hline Devoluciones & Sí permite con penalidad & $\begin{array}{c}\text { Permite sólo en tarifas altas y } \\
\text { con penalidad }\end{array}$ \\
\hline $\begin{array}{c}\text { Ratios entre mínimo y } \\
\text { máximo }\end{array}$ & $\begin{array}{c}\text { Bajo ratio entre precio } \\
\text { mínimo y máximo. }\end{array}$ & $\begin{array}{c}\text { Alto ratio entre precio } \\
\text { mínimo y precio máximo }\end{array}$ \\
\hline
\end{tabular}

Tabla I: Comparación de regulaciones entre las aerolíneas de bajo costo y modelos tradicionales. Fuente y elaboración propias.

Esta nueva dinámica en el mercado peruano representa un desafío, dado que existe el riesgo de eliminar la regulación tarifaria mínima de estadía a los clientes con alta disposición de pago, que valoran esta flexibilidad y comprarán los pasajes a un precio bajo. Sin embargo, los ingresos monetarios incrementales derivados de la estimulación de la demanda atribuida a la ganancia en términos de participación de mercado servirán como indicador del nivel relativo de éxito de esta práctica de gestión de ingresos. En consecuencia, al desregular la tarifa de mínimo de estadía, se espera tener ingresos incrementales positivos.

2. Marco teórico. -La gestión de ingresos es una práctica que ha nacido y evolucionado en la industria aeronáutica. American Airlines la aplicó al intentar recuperar la demanda perdida por la aparición de aerolíneas que ofrecían pasajes a precios muy bajos; la empresa logró determinar que la demanda variaba según criterios tales como la ruta, día de la semana, hora de vuelo y temporada; de esta forma se propicia que cada vuelo tenga una necesidad particular de asientos asignados a tarifas descontadas [5]. En 1985, la competitividad de American Airlines fue amenazada por la compañía People Express; en respuesta, American desarrolló un programa de gestión de ingreso monetario basado en la diferenciación de precios entre viajeros de placer y de negocios. Este programa incluía un sistema de "gestión de rendimiento" que utilizó algoritmos de optimización para determinar el número de asientos que permitiera proteger las futuras reservas de pasajeros con tarifa completa en cada vuelo, simultáneamente aceptaba a los pasajeros con tarifas bajas y pronta reserva. La gestión de ingresos de American Airlines fue reconocida como una aplicación estratégica importante de la ciencia administrativa [6]. American Airlines, definió la gestión de ingresos en sus inicios como la práctica que busca "vender el asiento correcto, al cliente correcto, al precio correcto" [7]. Su objetivo es la optimización del inventario de asientos, que es fijo y perecible, a través de decisiones tácticas en el corto plazo.

El modelo RM comprende establecer precios y asignar productos complementarios o sustitutos enfocándose en la gestión de la demanda y es considerada como un complemento a la gestión cadena de suministro, con relación a las decisiones de suministro y los procesos de una empresa con el fin de reducir costos de producción y entrega [4]. El modelo se basa en un planeamiento para diversas industrias y 
particularmente en aerolíneas de transporte de pasajeros, cuyo objetivo es maximizar los ingresos monetarios a través de un planeamiento estratégico sostenible y dirigido a clases de tarifas. El modelo RM es aplicable con las siguientes condiciones [13]: 1) El vendedor -valga la redundancia- vende un stock fijo de capacidad perecedera; 2) Los pasajeros realizan reservas antes de la salida; 3) El vendedor maneja un conjunto de clases de tarifas, donde cada una tiene un precio fijo (al menos a corto plazo); 4) El vendedor puede cambiar la disponibilidad de las clases de tarifas a lo largo del tiempo. El modelo facilita establecer y actualizar la disponibilidad de clases de tarifas, donde cada una tiene un precio asociado que permanece constante durante el período de reserva. Gracias al éxito de aplicar el modelo RM en las aerolíneas, ha sido posible su aplicación en otras industrias tales como hoteles, alquiler de automóviles, transporte de mercancías y líneas de cruceros, muchas de las cuales usan los mismos (o similares) sistemas de reserva que las aerolíneas de pasajeros [9].

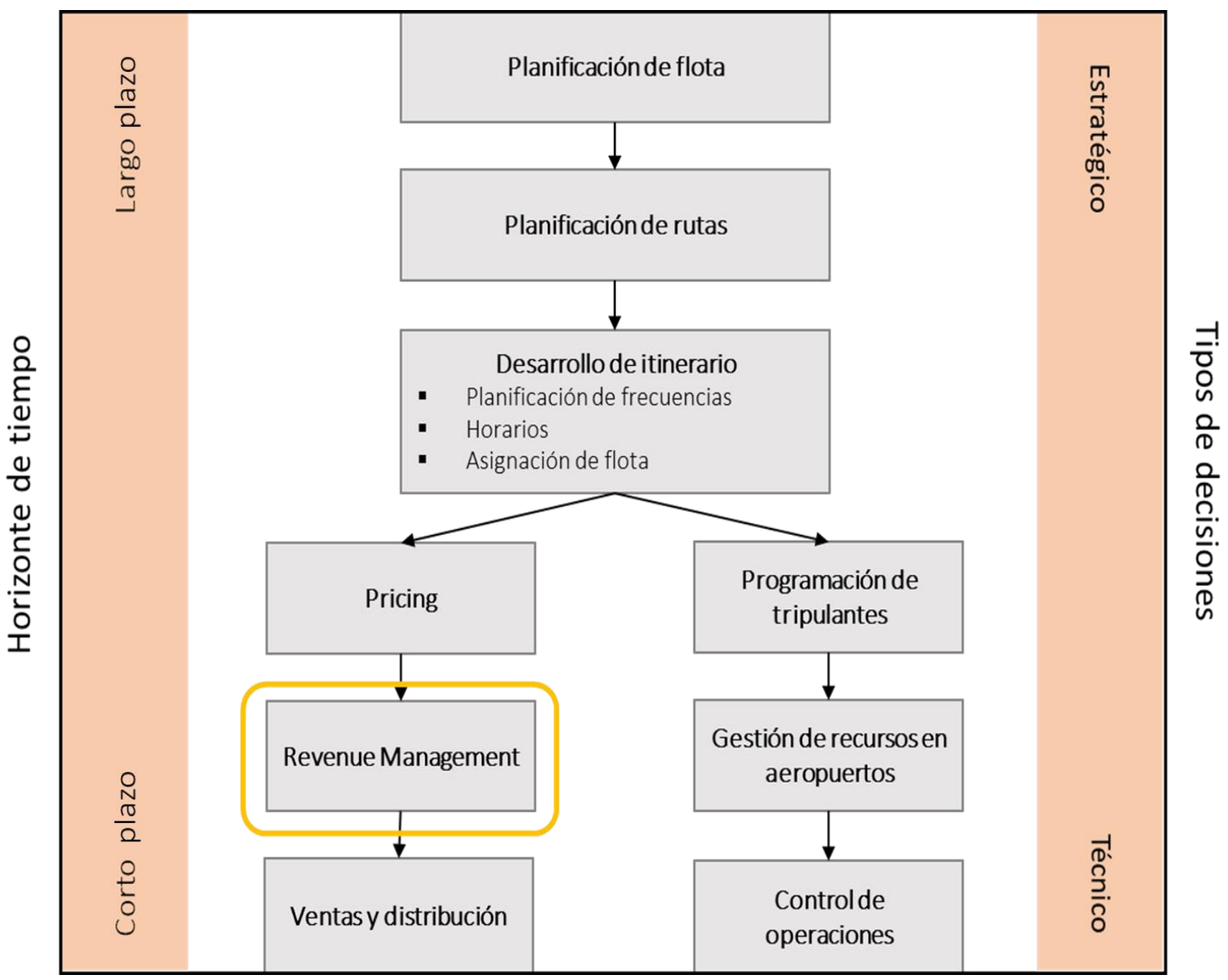

Figura III: Pasos en la gestión de una aerolinea. Fuente: Airline Schedule Optimization [8]

La fijación de tarifas de las aerolíneas de bajo costo, en general sigue un esquema general agresivo de competencia en precios, como resultado de la política actual de gestión de ingresos [13]. El precio es una respuesta adaptativa de la política de gestión de ingresos que se ajusta estratégicamente para aprovechar la segmentación de la demanda y maximizar los beneficios. Todo esto sin desviar la filosofía de mantener simple su sistema tarifario. En consecuencia, la estrategia de gestión de ingresos identifica segmentos de clientes estableciendo productos y servicios dirigidos a los mismos. Un elemento importante de la estrategia es la distinción entre los clientes de ocio y los comerciales identificados por las aerolíneas. Las aerolíneas aprovecharon este 
conocimiento y lo utilizaron para segmentar su mercado y crear productos virtuales orientados a los diferentes segmentos [9].

Esfuerzos computacionales se han realizado para aproximarse a la política óptima de gestión de ingresos. En la industria aeronáutica, la demanda ha sido evaluada a través de distintos modelos matemáticos [16] y estadísticos [15] para entender su comportamiento [13]. También se han empleado estrategias de predicción de capacidad, demanda y precios [17]. Debido a la complejidad de la competencia en la industria, muchas aproximaciones añaden a la modelación supuestos de competencia vertical y horizontal [18] y alianzas empresariales mediante aplicaciones de teoría de juegos [16] [19]. Adicionalmente se incluyeron otro grupo de supuestos sobre la naturaleza estocástica [15] [20] [21] y determinística [19] de los procesos. Por otro lado, algoritmos de optimización basados en el aprendizaje profundo reforzado constituyen la actual frontera de conocimientos usada por American Airlines [14]; esta aproximación es estadística y emplea un modelo que se entrena con datos simulados para realizar la optimización de forma automática con datos reales. Talluri y Van Ryzin (2005) [4] proponen novedosamente que, para un modelamiento más preciso de la demanda, es recomendable dividirla y agruparla en segmentos según sus preferencias y disposición de pago. Predecir el comportamiento de la demanda a partir de un segmento más homogéneo es más apropiado que hacerlo del universo total. De esta manera se conoce las características de este grupo y se puede ofrecer un producto que se acomode a sus necesidades.

El concepto de "Pricing" nace de la interpretación del correcto uso del producto, que se basa en encontrar la estrategia de precios adecuada para cada segmento de mercado identificado, a partir del modelo de demanda agregada. Desde el momento que se realiza la disposición de pago se da origen a una estructura tarifaria, por la agrupación de distintos productos. Esta diversidad de productos permite maximizar ingresos abarcando la mayor cantidad de puntos dentro de la curva de demanda. Phillips [9] afirma que, para muchas organizaciones, el precio incluye un conjunto de decisiones complejas. Si bien la mayoría de las empresas tienen conocimiento acerca de los precios de lista que han establecido para sus productos, no suelen conocer con claridad los precios que realmente pagan los clientes debido a que frecuentemente aplican en simultáneo diferentes descuentos, ajustes y rebajas a cada venta. Por esta razón, es fundamental distinguir entre el precio de lista de un bien y el precio final pagado por el cliente. El precio de lista es genérico, mientras que el precio final puede ser diferente para cada cliente. Aquellas aerolíneas que deseen ser competitivas deben adoptar las condiciones de regulación tarifaria. Sin embargo, es posible que esta acción genere una guerra de precios en donde el valor de mercado caiga y el mayor beneficiario sea el pasajero, esto se conoce como "Spiral-down". Este efecto sucede cuando interpretaciones o suposiciones erróneas del comportamiento del pasajero producen que, ventas de boletos con tarifas altas, así como los niveles de protección e ingreso monetario se reduzcan de manera sistemática. El espiral es resultado de una falla en la gestión de ingresos donde existe una mayor disponibilidad de pasajes de tarifa baja y menor nivel en ventas de tarifas altas, entonces se forma el patrón de descenso en espiral [10].

Adicionalmente, las aerolíneas pueden gestionar también el concepto llamado "Capacity", el cual permite asignar una cantidad de asientos por la cantidad esperada de pasajeros en cada nivel de tarifa específico. Este concepto permite realizar un cálculo de costos exacto, incluyendo los costos de operación individual para aviones y vuelos, así como de asiento disponible por kilómetro [11]. La combinación de "Pricing" y "Capacity" busca, a través de identificar los precios que los pasajeros están dispuestos a

Memoria Investigaciones en Ingeniería, 2019. $\mathrm{N}^{\circ} 17(1) . p p$ 61-71

https://doi.org/10.36561/ING.17.4

ISSN 2301-1092 • ISSN (en línea) 2301-1106 
pagar, maximizar ingresos monetarios priorizando espacios para aquellos pasajeros con mayor disposición de pago y capturando a aquellos más sensibles al precio. El rol principal que tiene la mejora de los aeropuertos en el desarrollo de las ciudades es permitir una mayor conectividad entre mercados laborales internacionales o regionales. No solo el comercio de bienes es importante en términos de competitividad global y sostenibilidad local [23], sino la movilidad de las personas que dinamiza el trabajo y permite una mayor división de este [24], así como una aproximación cultural e inclusiva entre ciudades lejanas [25].

\section{Caso de negocio.}

3.1. Planteamiento del problema. -En los últimos años se ha podido observar la entrada de aerolíneas con un nuevo modelo de bajo costo modificando el comportamiento en la industria aeronáutica mundial. Las aerolíneas de bajo costo tienen un modelo simplificado en cuanto a niveles y regulaciones en las tarifas, dificultando la competitividad del modelo tradicional de las aerolíneas. Como consecuencia, la disposición de pago en segmentos de alto valor ha disminuido, así mismo, la flexibilidad y el nivel de servicio ya no son suficientes para atraer a los clientes. Esto se evidencia en el bajo nivel de ocupación de asientos con tarifas altas, lo cual indica que la demanda de pasajeros corporativos se contrae. En la figura 4 se representa la caída de la curva de utilidad de la demanda, donde la disposición de pago se mantiene, pero la cantidad de pasajeros dispuestos a pagar los niveles tarifarios establecidos disminuye. Frente a este contexto, se considera la eliminación de restricciones tarifarias como técnica para alcanzar mayor competitividad esperando capturar a los clientes con mayor flexibilidad. A nivel global, esto ha representado un gran desafío, dado que existe el riesgo de que al eliminar las restricciones en las estructuras tarifarias los clientes no mantengan el tipo de comportamiento esperado, de acuerdo a la segmentación en la cual se les agrupó, y que, por el contrario, tomen el primer precio disponible.

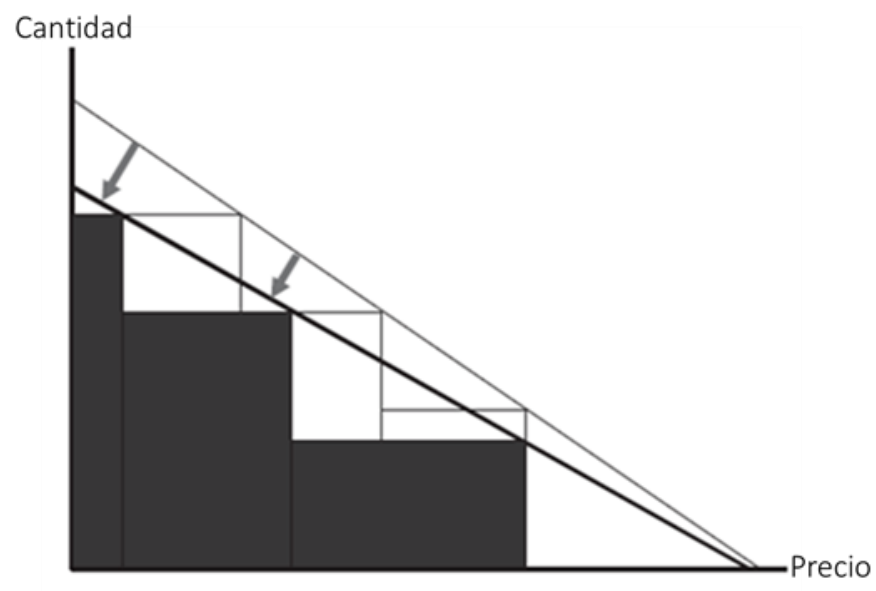

Figura IV: Contracción de demanda de segmentos con alta disposición de pago [12]

Dada la coyuntura competitiva entre aerolíneas tradicionales en la industria peruana, una de las empresas que opera a nivel nacional e internacional, ha optado por explorar e implementar la propuesta mencionada en este artículo; permitiendo comprobar que, eliminar las restricciones de mínimo de estadía, incrementa la competitividad e incentiva el crecimiento de pasajeros en la industria.

4. Metodología. -Con el fin de experimentar la eliminación de la regulación de mínimo de estadía en las tarifas de una aerolínea tradicional, este estudio sugiere los 
siguientes pasos: a) Planteamiento de objetivos; b) hipótesis; c) parámetro de medición; d) resultados; y e) conclusiones. De esta forma se busca mantener un orden que permita identificar los efectos generados y obtener conclusiones positivas o negativas con relación a ingresos monetarios.

El objetivo principal es identificar el efecto de la implementación mediante la comparación reflexiva entre el tipo de demanda de una estructura de precios de pasaje pre-implementación con la respectiva estructura de demanda de pasajes post implementación (ver Tabla II). Los objetivos secundarios implican, en primer lugar, analizar los fenómenos de estimulación y dilución de acuerdo al comportamiento de compra del pasajero y la tarifa que adquiere. Segundo, analizar y determinar si el cambio producido en estos fenómenos es producto de la implementación o también es influenciada por otros factores.

\begin{tabular}{|c|c|c|c|c|}
\hline \multirow{2}{*}{ Nivel tarifario } & \multicolumn{2}{|c|}{ Estadía } & \multicolumn{2}{c|}{ Anticipación } \\
\cline { 2 - 5 } & Pre medida & Post medida & Pre medida & Post medida \\
\hline S1 & Ninguna & Ninguna & Ninguna & Ninguna \\
\hline S2 & Ninguna & Ninguna & 3 días & 3 días \\
\hline S3 & 2 días & Ninguna & 7 días & 7 días \\
\hline S4 & 3 días & Ninguna & 14 días & 14 días \\
\hline S5 & 4 días & Ninguna & 30 días & 30 días \\
\hline
\end{tabular}

Tabla II: Estructura tarifaria con regulaciones de estadía y anticipación para un periodo Pre y Post implementación. Fuente: Elaboración propia

En relación a la hipótesis se declara esperar los siguientes comportamientos:

- Dilución: Pasajeros que valoran comprar pasajes sin mínimo de estadía pagando la tarifa base si es que la encuentran disponible.

- Estimulación o ganancia de participación de mercado: Existen pasajeros que valoran comprar pasajes sin mínimo de estadía y no encuentran tarifas que se acomoden a su disposición de pago por lo cual escogen otras aerolíneas.

La hipótesis formulada que existen ganancias monetarias considerables después de la implementación; además, sucede en paralelo con una dilución de pasajeros que previamente pagaban tarifas altas pero que actualmente escogen la tarifa baja desregulada; Finalmente, esta desregulación tarifaria captura una parte del mercado de pasajeros que demandan pasajes sin límite de estadía, pero no compraban en la aerolínea estudiada por no encontrar tarifas que se acomoden a su disposición de pago. Las ganancias monetarias se deben a distintos factores que estimularon la demanda en el periodo de estudio, el cual comprende la comparación reflexiva (como el crecimiento económico, shocks exógenos de demanda, publicidad, entre otros); sin embargo, hubo un efecto claro en el cambio en la estructura de demanda de tarifas de vuelo que probablemente se deba a la implementación del piloto.

Para medir los efectos de dilución y estimulación en primer lugar se realizó una segmentación de los pasajeros de la ruta considerando su comportamiento de estadía y la tarifa adquirida. Dentro del comportamiento de estadía se diferenció a aquellos que no ocupaban estadía de los que sí, y en cuanto a tarifa adquirida se diferenció a quienes compraban tarifa base de a los que compraban cualquier tarifa más alta. Con esta segmentación se distribuyó en cuatro cuadrantes. En ellos se podía encontrar a (1) pasajeros que no ocupaban estadía y compraban tarifa base (antes de la implementación de esta opción no estaba disponible), (2) pasajeros que no ocupaban estadía y compraban una tarifa más cara, (3) pasajeros que ocupaban estadías de 1 día a más y pagaban tarifas 
base y (4) pasajeros que ocupaban estadías de 1 día a más y pagaban tarifas altas. En los cuatro grupos se sumó la información de cantidad de pasajeros y se mostró la composición en términos porcentuales de la cantidad total de pasajeros transportados.

5. Resultados. -El estudio se implementó en dos rutas de Perú que transportan aproximadamente 50 mil pasajeros comerciales al mes, a través de una aerolínea internacional con operaciones en Latinoamérica. El resultado arrojó un incremento en la cantidad de pasajeros de $13 \%$ en el periodo de prueba versus el periodo anterior, lo que se arrojó un promedio de 63 mil pasajeros al mes. Se identificó cambio de comportamiento en cada uno de los cuadrantes (ver Tabla III). Los pasajeros que no ocupaban estadía aumentaron 12 puntos porcentuales respecto al periodo anterior. Esto se refleja en la composición del grupo de quienes no ocupan estadía, sin tener en cuenta la tarifa que pagaron, que pasó de representar un $13 \%$ a $25 \%$ del total de pasajeros. La composición de los pasajeros que compraban tarifas altas, sin diferenciar estadía, cae 6 puntos porcentuales respecto al periodo previo. Esto se traduce en una dilución de -235 mil USD considerando la menor composición de este segmento por la tarifa promedio que dejan estos pasajeros.

Dado que se abre la regulación y se permite comprar desde tarifas base, el cuadrante (1) de pasajeros que compran tarifas base sin ocupar estadía aumenta. A estos pasajeros se les considera como demanda frustrada, que antes no volaba con la aerolínea porque no encontraba el precio que estaba dispuesto a pagar teniendo en cuenta las condiciones de estadía. Esta mayor composición, un total que ha crecido, genera $230 \mathrm{mil}$ USD por estimulación. Los pasajeros que ocupaban estadías mayores a 1 día y compraban tarifas altas, lo hacen porque son las tarifas que encuentran disponibles y se acomodan a lo que están dispuestos a pagar, no muestran sensibilidad al atributo de estadía, por el contrario, pueden estar más enfocados en la anticipación con que compran u otras condiciones de las regulaciones tarifarias que no fueron modificadas. Mantener a estos pasajeros en su nivel tarifario permitió incrementar las ventas en 75 mil USD adicionales. En ingresos totales se calculó un aporte de 70 mil USD, lo que representa una mejora de $3 \%$ de los ingresos de la ruta con un incremento de $13 \%$ de pasajeros totales.

Pre medida

\begin{tabular}{|c|c|c|c|c|c|c|c|c|c|}
\hline & \multicolumn{8}{|c|}{ Comportamiento de estadia del pasajero } & \multirow[b]{2}{*}{ TOTAL } \\
\hline $\begin{array}{c}\text { Nivel } \\
\text { tarifario }\end{array}$ & OW & 0 & 1 & 2 & 3 & 4 & 5 & NS & \\
\hline S1 & $7 \%$ & $1 \%$ & $3 \%$ & $1 \%$ & $1 \%$ & $0 \%$ & $0 \%$ & $6 \%$ & $19 \%$ \\
\hline S2 & $2 \%$ & $0 \%$ & $0 \%$ & $0 \%$ & $0 \%$ & $0 \%$ & $0 \%$ & $2 \%$ & $6 \%$ \\
\hline S3 & $2 \%$ & $0 \%$ & $0 \%$ & $0 \%$ & $0 \%$ & $0 \%$ & $0 \%$ & $3 \%$ & $6 \%$ \\
\hline $\mathrm{S} 4$ & $1 \%$ & $0 \%$ & $0 \%$ & $0 \%$ & $0 \%$ & $0 \%$ & $0 \%$ & $10 \%$ & $13 \%$ \\
\hline S5 & $1 \%$ & $0 \%$ & $0 \%$ & $0 \%$ & $0 \%$ & $0 \%$ & $0 \%$ & $53 \%$ & $56 \%$ \\
\hline TOTAL & $13 \%$ & $1 \%$ & $3 \%$ & $3 \%$ & $3 \%$ & $2 \%$ & $0 \%$ & $73 \%$ & $100 \%$ \\
\hline
\end{tabular}

$\underline{\text { Post medida }}$

\begin{tabular}{|c|c|c|c|c|c|c|c|c|c|}
\hline & \multicolumn{8}{|c|}{ Comportamiento de estadía del pasajero } & \multirow[b]{2}{*}{ TOTAL } \\
\hline $\begin{array}{c}\text { Nivel } \\
\text { tarifario }\end{array}$ & OW & 0 & 1 & 2 & 3 & 4 & 5 & NS & \\
\hline S1 & $4 \%$ & $0 \%$ & $1 \%$ & $1 \%$ & $1 \%$ & $0 \%$ & $0 \%$ & $5 \%$ & $13 \%$ \\
\hline S2 & $2 \%$ & $0 \%$ & $0 \%$ & $0 \%$ & $0 \%$ & $0 \%$ & $0 \%$ & $3 \%$ & $6 \%$ \\
\hline S3 & $3 \%$ & $0 \%$ & $1 \%$ & $0 \%$ & $0 \%$ & $0 \%$ & $0 \%$ & $4 \%$ & $9 \%$ \\
\hline S4 & $4 \%$ & $0 \%$ & $1 \%$ & $1 \%$ & $0 \%$ & $0 \%$ & $0 \%$ & $8 \%$ & $14 \%$ \\
\hline S5 & $12 \%$ & $0 \%$ & $1 \%$ & $2 \%$ & $1 \%$ & $1 \%$ & $0 \%$ & $39 \%$ & $58 \%$ \\
\hline TOTAL & $25 \%$ & $2 \%$ & $4 \%$ & $4 \%$ & $3 \%$ & $2 \%$ & $1 \%$ & $60 \%$ & $100 \%$ \\
\hline
\end{tabular}

Tabla III: Matriz de comportamiento de estadía del pasajero según nivel tarifario para mercado nacional en periodos Pre y Post eliminación de regulación de mínimo de estadía. Fuente: Elaboración propia. 
6. Conclusiones. -El modelo de "gestión de ingresos" es una práctica estratégica que aporta mayor rentabilidad a una aerolínea. Está comprobado que en un entorno de crecimiento industrial donde operan muchas aerolíneas, se puede utilizar este modelo para modificar la composición de la demanda y proteger simultáneamente la industria de una guerra de precios aportando soluciones alternativas como la búsqueda de competitividad a partir de flexibilización, tal y como se demostró en el caso de estudio. Los nuevos pasajeros que obtuvo la aerolínea generaron ingresos incrementales que superaron la dilución de los pasajeros antiguos quienes compraron también en tarifas base, según la comparación reflexiva. Sin embargo, es necesario aislar el efecto de otros factores que estimulen la demanda. Adicionalmente, esto puede variar por la cantidad de cupos que se asignan a la tarifa base $\mathrm{u}$ otras características en el comportamiento del cliente.

Existen pasajeros en la industria que valoran comprar pasajes sin mínimo de estadía resultando aparentemente positivo el incremento de la competitividad por la eliminación de la regulación tarifaria de mínimo de estadía, dado que los pasajeros valoran este atributo y muestran sensibilidad al precio de acuerdo al cambio en la composición de la demanda. Esto se ilustra al habilitar tarifas base sin mínimo de estadía resultando el incremento absoluto de pasajeros. La dilución es generada al habilitar tarifas base para quienes valoraban el atributo estadía menor a 1 día, quienes estaban dispuestos a pagar una tarifa mayor, pero eligieron la más baja ahora disponible. Así mismo, existe un segmento de pasajeros que no se diluye porque su comportamiento está segmentado con otras regulaciones tarifarias que no fueron estudiadas en este documento.

En síntesis, este modelo de gestión de ingresos, de aplicarse en distintas aerolíneas tradicionales en el mercado peruano, puede generar considerables ingresos incrementales; sin embargo, se debe hacer énfasis en la mejora de competitividad obtenida respecto a las aerolíneas de bajo costo, en un entorno donde cada vez estas últimas son más numerosas. La contribución del modelo es socialmente importante porque, según la información sobre operadores de ruta, algunas regiones solo están conectadas por aerolíneas tradicionales, lo que impactaría en la mejora de competitividad y productividad aportando a la integración económica entre regiones.

\section{Referencias}

[1] Asociación Internacional de Transporte Aéreo, La importancia del transporte aéreo en Perú, Oxford Economics Report, 2016.

[2] Portal de turismo, Transporte aéreo en Perú genera 280 mil empleos y aporta US\$ 4.400 millones al PBI, 2017.

[3] Gestión, Transporte aéreo nacional de pasajeros en Perú creció 8.5\% en 2017, 2017.

[4] Talluri, K. T., \& Van Ryzin, G., The theory and practice of Revenue Management, 2005, New York: Springer.

[5] García, F., Revenue management: maximización de ingresos de las aerolíneas, 2016, México.

[6] Anderson, C. K., P. Bell, and S. P. Kaiser., Strategic operations research and the Edelman Prize finalist applications 1989 -1998, Operations Research, 2003, 52(1): p. 1- 13.

[7] Smith, B., Leimkuhler, J., \& Darrow, R., Yield Management at American Airlines. Interfaces. 1992. 22(1): p. 8-31.

[8] Barnhart, C., Airline Schedule Optimization, The Global Airline Industry, 2009, London: John Wiley \& Sons.

[9] Phillips, R. Pricing and Revenue Optimization, 2005, Stanford University Press.

[10] Cooper, W. L., Homem-de-Mello, T., \& Kleywegt, A. J. Models of the Spiral-Down Effect in Revenue Management, Operations Research. 2006, 54(5): p. 968-987.

[11] Tsa, W.-H., \& Kuo, L., Operating costs and capacity in the airline industry. Journal of Air Transport Management, 2004, 10(4): p. 269-275.

Memoria Investigaciones en Ingeniería, 2019. $\mathrm{N}^{\circ} 17(1) . p p$ 61-71

https://doi.org/10.36561/ING.17.4

ISSN 2301-1092 • ISSN (en línea) 2301-1106 
[12] Belobaba, P. P., Fundamentals of Pricing and Revenue Management, In P. P. Belobaba, A. Odoni, \& C. Barnhart, The global airline industry, 2009, p. 84-87, UK: John Wiley and Sons.

[13] Alderighi, M. et al. (2019). Is low-cost carriers' revenue management a firm capability? Journal of Air Transport Management, 78, 15-22.

[14] Mohd, S. et al. (2019). Autonomous Airline Revenue Management: A Deep Reinforcement Learning Approach to Seat Inventory Control and Overbooking.

[15] Büsing, C., Kadatz, D., \& Cleophas, C. Capacity Uncertainty in Airline Revenue Management: Models, Algorithms, and Computations, 2019. Transportation Science, 53, 2, 383-400.

[16] Liu, M., Hou, Y., Chu, F., \& Huo, J. Revenue management of airline alliances with integral planning strategy, 2017. 14th International Conference on Service Systems and Service Management (ICSSSM) 1-6.

[17] Zaki, H. Forecasting for Airline Revenue Management, 2000. Journal of Business Forecasting Methods and Systems, 19, 2-7.

[18] Netessine, S., \& Shumsky, R. A. Revenue Management Games: Horizontal and Vertical Competition, 2005. Management Science, 51, 5, 813.

[19] Grauberger, W., \& Kimms, A. Revenue management under horizontal and vertical competition within airline alliances, 2016. Omega, 59, 228-237.

[20] Gao, J. \& Le, M. Mathematical programming of airline revenue management with passenger choice behavior, 2018. 14th International Conference on Natural Computation, Fuzzy Systems and Knowledge Discovery (ICNC-FSKD). 669-676.

[21] Arslan, A. M., Frenk, J. B. G., \& Sezer, S. O. On the single-leg airline revenue management problem in continuous time, 2015. Mathematical Methods of Operations Research, 81, 1, 27-52.

[22] Mohd, S. et al. Autonomous Airline Revenue Management: A Deep Reinforcement Learning Approach to Seat Inventory Control and Overbooking, 2019.

[23] Conventz, S., In Derudder, B., In Thierstein, A., \& In Witlox, F. Hub cities in the knowledge economy: Seaports, airports, brainports. 2016

[24] Appold, S. J., \& Kasarda, J. D. The airport city phenomenon: Evidence from large US airports, 2016. Urban Studies, 50, 1239-1259.

[25] Leeuw, P. Airport city development at mature airports: Structural, strategic and commercial aspects along the path of a massive change process, 2019. Journal of Airport Management, 13, 2, 122-132. 